\title{
On energetics and inertial-range scaling laws of two-dimensional magnetohydrodynamic turbulence
}

\author{
Luke A. K. Blackbourn and Chuong V. Tran $\dagger$ \\ School of Mathematics and Statistics, University of St Andrews, St Andrews KY16 9SS, UK \\ (Received 15 December 2011; revised 23 April 2012; accepted 26 April 2012; \\ first published online 12 June 2012)
}

We study two-dimensional magnetohydrodynamic turbulence, with an emphasis on its energetics and inertial-range scaling laws. A detailed spectral analysis shows that dynamo triads (those converting kinetic into magnetic energy) are associated with a direct magnetic energy flux while anti-dynamo triads (those converting magnetic into kinetic energy) are associated with an inverse magnetic energy flux. As both dynamo and anti-dynamo interacting triads are integral parts of the direct energy transfer, the anti-dynamo inverse flux partially neutralizes the dynamo direct flux, arguably resulting in relatively weak direct energy transfer and giving rise to dynamo saturation. This result is consistent with a qualitative prediction of energy transfer reduction due to Alfvén wave effects by the Iroshnikov-Kraichnan theory (which was originally formulated for magnetohydrodynamic turbulence in three dimensions). We numerically confirm the correlation between dynamo action and direct magnetic energy flux and investigate the applicability of quantitative aspects of the Iroshnikov-Kraichnan theory to the present case, particularly its predictions of energy equipartition and $k^{-3 / 2}$ spectra in the energy inertial range. It is found that for turbulence satisfying the Kraichnan condition of magnetic energy at large scales exceeding total energy in the inertial range, the kinetic energy spectrum, which is significantly shallower than $k^{-3 / 2}$, is shallower than its magnetic counterpart. This result suggests no energy equipartition. The total energy spectrum appears to depend on the energy composition of the turbulence but is clearly shallower than $k^{-3 / 2}$ for $r \approx 2$, even at moderate resolutions. Here $r \approx 2$ is the magnetic-to-kinetic energy ratio during the stage when the turbulence can be considered fully developed. The implication of the present findings is discussed in conjunction with further numerical results on the dependence of the energy dissipation rate on resolution.

Key words: MHD turbulence

\section{Introduction}

In incompressible magnetohydrodynamic (MHD) turbulence, the total (kinetic plus magnetic) energy is conserved and transferred from large to small scales (direct transfer). This is true for both two and three dimensions (2D and 3D), although the underpinning mechanisms responsible for this dynamical behaviour can be quite 
different in each case. In 3D, the usual vortex stretching mechanism can act unilaterally to promote and sustain a direct flux of kinetic energy. Meanwhile, the production of magnetic small scales by magnetic stretching provides a similar but separate route for a direct flux of magnetic energy. These mechanisms, together with the conversion of kinetic into magnetic energy (dynamo action) and the reverse process (referred to as anti-dynamo action) through the quadratic coupling between the velocity and magnetic fields via the Lorentz force, shape the picture of direct energy transfer in 3D. In 2D, on the other hand, the vortex stretching mechanism is absent. It follows that the large scales can rid themselves of kinetic energy only through the coupling between the velocity and magnetic fields. Hence, dynamos (a manifestation of magnetic stretching) and anti-dynamos play key roles in the energy transfer process. Given the quadratic nonlinear interactions - an apparent analogy with 3D Navier-Stokes turbulence - usual arguments based on Kolmogorov's phenomenology have been used to deduce the classical $k^{-5 / 3}$ spectrum in the energy inertial range for both 2D and 3D MHD turbulence. Note that in 2D, the magnetic potential is materially conserved and its variance is known to undergo an inverse transfer (Fyfe \& Montgomery 1976; Pouquet 1978; Biskamp \& Bremer 1993; Kim \& Dubrulle 2002). This conservation limits large-scale dynamo action and may favour dynamo saturation, which has been widely observed (cf. Tobias \& Cattaneo 2008; Cattaneo \& Tobias 2009) and appears to be an intrinsic dynamical property, especially for turbulence developed from weak seed magnetic fields.

The quadratic coupling between the velocity and magnetic fields can give rise to rich dynamical behaviour. However, this additional nonlinear interaction does not necessarily mean that 3D MHD turbulence is 'more turbulent' than its Navier-Stokes counterpart. In fact, the theory of Iroshnikov (1964) and Kraichnan (1965) (hereafter referred to as the IK theory) suggests the contrary (see also Moffatt 1967). More quantitatively, Kraichnan (1965) argues that when the magnetic energy in sub-inertial wavenumbers exceeds the total energy in the inertial range, the energy transfer can be reduced to a certain extent. This reduction is due to Alfvén waves, which intuitively undermine nonlinear effects. The reduction of energy transfer (reduction of nonlinear effects or suppression of turbulence) means that stronger excitation of the inertial range is required before the energy can be transferred to the dissipation scale, thereby allowing shallower spectra to develop (Tran, Blackbourn \& Scott 2011). Indeed, the IK theory predicts a $k^{-3 / 2}$ energy inertial range, which is slightly shallower than the classical Kolmogorov spectrum. Kraichnan (1965) further predicts energy equipartition in the inertial range, i.e. identical magnetic and kinetic energy spectra within this range. This prediction has found little support as there exists ample evidence for a clear mismatch between these spectra (Grappin, Pouquet \& Leorat 1983; Müller \& Grappin 2005; Podesta, Roberts \& Goldstein 2007; Boldyrev \& Perez 2009; Tessein et al. 2009). It is interesting to note that on the basis of local anisotropy, Sridhar \& Goldreich (1994) and Goldreich \& Sridhar (1995) dismiss the IK theory as incorrect.

There has been considerable debate in the literature over the scaling law in the energy inertial range of MHD turbulence. Thus far, this issue has not been resolved adequately. Since the inertial range has little energy (whether it be Kolmogorov or IK), one would expect the Kraichnan condition (magnetic energy at large scales in excess of total energy in the inertial range) to hold for a majority of numerical simulations and physical systems. In other words, one would expect the IK spectrum to be realizable for a majority of cases, even for those with weak magnetic seeds, where dynamo action can amplify the large-scale magnetic field significantly before becoming saturated. However, this does not appear to be the case. In fact, evidence has 
been presented for both Kolmogorov's and IK's spectra under conditions presumably in favour of the latter (Verma et al. 1996; Galtier, Pouquet \& Mangeney 2005; Ng et al. 2010; Beresnyak 2011). Furthermore, on the basis of numerical results for 3D MHD turbulence at unity magnetic Prandtl number $(P r=1)$, Lee et al. (2010) even suggested the realizability of the shock-dominated Burgers spectrum $k^{-2}$, together with the Kolmogorov and IK spectra.

In this study, we investigate 2D MHD turbulence both theoretically and numerically, focusing on its energetics and possible inertial-range scaling laws. A detailed spectral analysis shows that within a dynamo triad (one converting kinetic into magnetic energy), the smaller-scale magnetic mode gains energy at the expense of both the larger-scale magnetic mode and the mechanical mode. This means that dynamo action is associated with a direct magnetic energy flux. On the other hand, within an antidynamo triad (one converting magnetic into kinetic energy), the smaller-scale magnetic mode loses energy to both the larger-scale magnetic mode and the mechanical mode. This means that anti-dynamo action is associated with an inverse magnetic energy flux. The anti-dynamo inverse flux can partially neutralize the dynamo direct flux, presumably resulting in dynamo saturation and relatively weak direct energy transfer. This result is consistent with the qualitative aspect of the IK theory on energy transfer reduction by Alfvén wave effects. We numerically confirm the correlation between dynamo action and direct magnetic energy flux and investigate the applicability of the quantitative aspect of the IK theory to the present case. Our simulation results indicate no energy equipartition and unrealizability of the IK spectrum for turbulence satisfying the Kraichnan condition (magnetic energy at large scales exceeding total energy in the inertial range). More precisely, for turbulence at $\operatorname{Pr}=1$ decaying from a localized energy reservoir having fixed total energy and various magnetic-to-kinetic energy ratios $r_{0}=r(0)$ within the range $[1 / 16,16]$, it is found that when the turbulence becomes fully developed, the Kraichnan condition is satisfied across the board (with $r \in(2,5)$ ), owing to strong dynamo action for the low- $r_{0}$ cases. Yet the kinetic energy spectrum, which is significantly shallower than $k^{-3 / 2}$, is shallower than its magnetic counterpart, thus implying no energy equipartition. The total energy spectrum is shallower than $k^{-3 / 2}$ for the cases with $r \approx 2$ and appears to be as shallow as $k^{-3 / 2}$ (even at moderate resolutions) for higher $r$. This raises the question of the applicability of IK's theory to 2D MHD turbulence. We discuss the implication of the present results in conjunction with numerical evidence for the divergence of the time of peak energy dissipation in the small-diffusivity limit.

\section{Governing equations and conservation laws}

Consider an electrically conducting and incompressible fluid, which is subject to no external magnetic field or mechanical stirring. The equations governing the fluid motion and the internally generated magnetic field are

$$
\begin{gathered}
\boldsymbol{u}_{t}+(\boldsymbol{u} \cdot \nabla) \boldsymbol{u}+\nabla p=(\boldsymbol{b} \cdot \nabla) \boldsymbol{b}+v \Delta \boldsymbol{u}, \\
\boldsymbol{b}_{t}+(\boldsymbol{u} \cdot \nabla) \boldsymbol{b}=(\boldsymbol{b} \cdot \nabla) \boldsymbol{u}+\mu \Delta \boldsymbol{b}, \\
\nabla \cdot \boldsymbol{u}=0=\nabla \cdot \boldsymbol{b},
\end{gathered}
$$

where $\boldsymbol{u}(\boldsymbol{x}, t)$ is the fluid velocity, $\boldsymbol{b}(\boldsymbol{x}, t)$ is the magnetic field vector, $p(\boldsymbol{x}, t)$ is the total pressure, $v$ is the viscosity, and $\mu$ is the magnetic diffusivity. For simplicity, the fluid domain is assumed to have no boundary, being either a periodic box or the entire space with suitable decay conditions for $\boldsymbol{u}$ and $\boldsymbol{b}$ at infinity. Throughout this paper, $\langle\cdot\rangle$ denotes an integral over the domain or a domain average in the periodic case. In 
the momentum equation $(2.1)$, the term $(\boldsymbol{b} \cdot \nabla) \boldsymbol{b}$ represents the Lorentz force. In the induction equation (2.2), the stretching term $(\boldsymbol{b} \cdot \nabla) \boldsymbol{u}$ allows $\boldsymbol{b}$ to be amplified (at the expense of $\boldsymbol{u})$ by the fluid velocity gradients.

In the absence of viscosity and magnetic diffusivity, (2.1) and (2.2) possess a number of conservation laws. Most important is the conservation of the total energy $E=E_{\boldsymbol{u}}+E_{\boldsymbol{b}}=\left\langle|\boldsymbol{u}|^{2}\right\rangle / 2+\left\langle|\boldsymbol{b}|^{2}\right\rangle / 2$. This is readily seen from the kinetic and magnetic energy evolution equations

$$
\begin{aligned}
& \frac{1}{2} \frac{\mathrm{d}}{\mathrm{d} t}\left\langle|\boldsymbol{u}|^{2}\right\rangle=\langle\boldsymbol{u} \cdot(\boldsymbol{b} \cdot \nabla) \boldsymbol{b}\rangle-v\left\langle|\nabla \boldsymbol{u}|^{2}\right\rangle=\langle\boldsymbol{u} \cdot(\boldsymbol{b} \cdot \nabla) \boldsymbol{b}\rangle-\epsilon_{\boldsymbol{u}}, \\
& \frac{1}{2} \frac{\mathrm{d}}{\mathrm{d} t}\left\langle|\boldsymbol{b}|^{2}\right\rangle=\langle\boldsymbol{b} \cdot(\boldsymbol{b} \cdot \nabla) \boldsymbol{u}\rangle-\mu\left\langle|\nabla \boldsymbol{b}|^{2}\right\rangle=\langle\boldsymbol{b} \cdot(\boldsymbol{b} \cdot \nabla) \boldsymbol{u}\rangle-\epsilon_{\boldsymbol{b}},
\end{aligned}
$$

where the triple-product terms on the right-hand sides exactly cancel each other. These terms represent the conversion between kinetic and magnetic energy and play important roles in the turbulent dynamics. Another well-known invariant is the crosshelicity $\langle\boldsymbol{u} \cdot \boldsymbol{b}\rangle$. This, together with the conservation of total energy, further implies the conservation of $\left\langle\left|\boldsymbol{Z}^{ \pm}\right|^{2}\right\rangle$, where $\boldsymbol{Z}^{ \pm}=\boldsymbol{u} \pm \boldsymbol{b}$ are known as the Elsässer variables. When expressed in terms of these variables, the MHD equations take a symmetric form (cf. Kraichnan 1965), which is convenient for the study of Alfvén waves.

The energy dissipation rate $\epsilon(t)=\epsilon_{u}(t)+\epsilon_{b}(t)$ plays an important role in the phenomenological theory of turbulence. Recently, it has been used as a key parameter in mathematical studies to rule out (rigorously in $2 \mathrm{D}$ and semi-rigorously in 3D) spectra steeper than $k^{-5 / 3}$ if the global maximum dissipation rate, say $\epsilon_{T}=\epsilon(T)$, does not vanish as a power law in $v$ in the limit $v=\mu \rightarrow 0$ (Tran \& Blackbourn 2012; Tran $\& \mathrm{Yu} 2012$ ). Owing to global regularity of solutions in $2 \mathrm{D}$ (for both $\nu>0$ and $\mu>0$ ), $\epsilon(t)$ is bounded and the existence of $\epsilon_{T}$ is guaranteed. However, the detailed behaviour of $\epsilon_{T}$ and $T$ (both in general depend on $v$ ) is not known with certainty in the inviscid and diffusionless limit. Nonetheless, evidence for finite and viscosity-independent $\epsilon(t)$ has been presented for both 2D (Biskamp \& Welter 1989; Politano, Pouquet \& Sulem 1989) and 3D (Mininni, Pouquet \& Montgomery 2006; Lee et al. 2010). Here our 2D numerical results (see $\S 4$ ) suggest that $T$ slowly (probably logarithmically) diverges while $\epsilon_{T}$ equally slowly converges. It is, however, unclear whether or not $\epsilon_{T}$ tends to a non-zero constant.

In two dimensions, (2.1) and (2.2) can be conveniently written as

$$
\begin{gathered}
\frac{\partial \omega}{\partial t}+J(\psi, \omega)=J(a, \Delta a)+v \Delta \omega, \\
\frac{\partial a}{\partial t}+J(\psi, a)=\mu \Delta a,
\end{gathered}
$$

where $\psi$ is the streamfunction, $\omega=\Delta \psi$ is the fluid vorticity, $a$ is the magnetic potential and $J(\cdot, \cdot)$ denotes the Jacobian. The incompressible velocity $\boldsymbol{u}$ and nondivergent magnetic field $\boldsymbol{b}$ are given, respectively, in terms of $\psi$ and $a$ by $\boldsymbol{u}=\left(-\psi_{y}, \psi_{x}\right)$ and $\boldsymbol{b}=\left(a_{y},-a_{x}\right)$. The current $j$ is given by $j=-\Delta a$. The energy and cross-helicity are given by $E=\left\langle|\nabla \psi|^{2}\right\rangle / 2+\left\langle|\nabla a|^{2}\right\rangle / 2$ and $\langle\boldsymbol{u} \cdot \boldsymbol{b}\rangle=-\langle\nabla \psi \cdot \nabla a\rangle$. As mentioned above, $a$ is materially conserved (when $\mu=0$ ) and its variance $\left\langle a^{2}\right\rangle$ is transferred to the large scales. Note that $a$ is an active scalar, feeding back on the velocity field through the Lorentz force. In the absence of an external field, $\boldsymbol{a}$ (and $\boldsymbol{b}$ ) eventually decays to zero, even in the presence of a persistent injection of kinetic energy (Pouquet 1978). 
The simultaneous conservation of the kinetic energy and mean-square vorticity by the advection term $J(\psi, \omega)$, owing to $\langle\psi J(\psi, \omega)\rangle=0=\langle\omega J(\psi, \omega)\rangle$, is known to give rise to an inverse transfer of kinetic energy in the non-conducting case. This inverse transfer mechanism operates on a relatively slow time scale and limited spectral extent (Tran \& Dritschel 2006; Dritschel et al. 2008) and, in the present case, remains active. One would therefore expect some inverse flow of kinetic energy although this flow could be partially or completely cancelled out by the 'more vigorous' direct transfer of the total energy. In any case, a spectral peak of kinetic energy is expected to spread out in both directions (predominantly to higher wavenumbers though) relatively quickly, a fact readily confirmed by the numerical results in $\S 4$.

Let $\widehat{\psi}_{\boldsymbol{k}}(t)$ and $\widehat{a}_{\boldsymbol{k}}(t)$ denote, respectively, the Fourier transforms of $\psi(\boldsymbol{x}, t)$ and $a(\boldsymbol{x}, t)$, where $\boldsymbol{x}=(x, y)$ and $\boldsymbol{k}=\left(k_{x}, k_{y}\right)$. The reality of both $\psi(\boldsymbol{x}, t)$ and $a(\boldsymbol{x}, t)$ requires $\widehat{\psi}_{\boldsymbol{k}}(t)=\widehat{\psi}_{-k}^{*}(t)$ and $\widehat{a}_{\boldsymbol{k}}(t)=\widehat{a}_{-\boldsymbol{k}}^{*}(t)$, where the asterisk denotes a complex conjugate. In terms of $\widehat{\psi}_{\boldsymbol{k}}(t)$ and $\widehat{a}_{\boldsymbol{k}}(t), \psi(\boldsymbol{x}, t)$ and $a(\boldsymbol{x}, t)$ can be represented by

$$
\begin{aligned}
\psi(\boldsymbol{x}, t) & =\sum_{k} \widehat{\psi}_{k}(t) \exp \{\mathrm{i} \boldsymbol{k} \cdot \boldsymbol{x}\}, \\
a(\boldsymbol{x}, t) & =\sum_{\boldsymbol{k}} \widehat{a}_{\boldsymbol{k}}(t) \exp \{\mathrm{i} \boldsymbol{k} \cdot \boldsymbol{x}\} .
\end{aligned}
$$

The equations governing the evolution of $\widehat{\psi}_{k}(t)$ and $\widehat{a}_{k}(t)$ are

$$
\begin{aligned}
\frac{\mathrm{d}}{\mathrm{d} t} \widehat{\psi}_{k} & =\sum_{\ell+\boldsymbol{m}=\boldsymbol{k}} \frac{m^{2}-\ell^{2}}{k^{2}} \boldsymbol{\ell} \times \boldsymbol{m}\left(\widehat{a}_{\ell} \widehat{a}_{m}-\widehat{\psi}_{\ell} \widehat{\psi}_{m}\right), \\
\frac{\mathrm{d}}{\mathrm{d} t} \widehat{a}_{k} & =\sum_{\ell+\boldsymbol{m}=k} \boldsymbol{\ell} \times \boldsymbol{m} \widehat{\psi}_{\ell} \widehat{a}_{\boldsymbol{m}},
\end{aligned}
$$

where the dissipation terms have been omitted and the sum is over all possible wavevectors satisfying the indicated triad condition. Here $k=|\boldsymbol{k}|, \ell=|\ell|, m=|\boldsymbol{m}|$, and $\ell \times \boldsymbol{m}=\ell_{x} m_{y}-\ell_{y} m_{x}$.

\section{Dynamo and energy transfer}

This section presents some simple yet illuminating arguments concerning dynamos and direct energy transfer. As in the introductory section, the respective terms 'dynamo' and 'anti-dynamo' refer to a conversion from kinetic to magnetic energy and the reverse process (also known as magnetic reconnection). This somewhat liberal use of terminology is for convenience and poses little risk of confusion with the conventional meaning of dynamo in the context of 3D MHD turbulence.

The dual conservation of the magnetic potential variance $\left\langle a^{2}\right\rangle$ and total energy $E$ imposes a stiff constraint on dynamo action. Consider turbulence developed from a spectrally localized magnetic energy reservoir around some wavenumber $k_{*}$. No requirement for the spectral distribution of the kinetic energy reservoir is necessary for the present argument. The conservation of $\left\langle a^{2}\right\rangle$ allows no significant dynamo action to take place at large scales $\left(k \leqslant k_{*}\right)$ as that would entail the production of a relatively large amount of $\left\langle a^{2}\right\rangle$, thereby violating its conservation. On the other hand, dynamo action occurring towards the small scales $\left(k \geqslant k_{*}\right)$ is permitted, and in fact, plausible by magnetic stretching. However, there is a natural limitation to small-scale dynamo action. Since the induction equation is linear, exponential growth of $E_{b}$ is possible. Now owing to the conservation of $E$, such a growth is short-lived for limited 
$E_{u}$ available. As the kinetic energy reservoir becomes increasingly depleted, dynamo action necessarily becomes saturated. In $\S 4$, the results from numerical simulations suggest that saturation is achieved through progressively weaker cycles of dynamo and anti-dynamo action.

The triple-product terms representing the conversion between kinetic and magnetic energy are linear in $\psi$ and quadratic in $a$. In wavenumber space, this conversion occurs in wavenumber triads, each individually conserving the total energy and consisting of one mode from $\psi$ (mechanical mode) and two modes from $a$ (magnetic modes). Given that the dynamics of usual (non-magnetic) 2D turbulence and scalar advection are relatively well-understood, the behaviour of these triads holds the key to our understanding of 2D MHD turbulence. The remainder of this section examines in detail the dynamics of these triads.

Consider an interacting triad $\widehat{\psi}_{\boldsymbol{k}}(t) \exp \{\mathrm{i} \boldsymbol{k} \cdot \boldsymbol{x}\}, \widehat{a}_{\boldsymbol{\ell}}(t) \exp \{\mathrm{i} \boldsymbol{\ell} \cdot \boldsymbol{x}\}$, and $\widehat{a}_{\boldsymbol{m}}(t) \exp \{\mathrm{im} \cdot \boldsymbol{x}\}$, where $\boldsymbol{k}=\boldsymbol{\ell}+\boldsymbol{m}$. Within this triad, the equations governing the energy conversion (and transfer) are

$$
\begin{aligned}
\frac{\mathrm{d}}{\mathrm{d} t} k^{2}\left|\widehat{\psi}_{k}\right|^{2} & =\left(\ell^{2}-m^{2}\right) \boldsymbol{l} \times \boldsymbol{m}\left(\widehat{\psi}_{k}^{*} \widehat{a}_{\ell} \widehat{a}_{m}+\widehat{\psi}_{k} \widehat{a}_{\ell}^{*} \widehat{a}_{m}^{*}\right), \\
\frac{\mathrm{d}}{\mathrm{d} t} \ell^{2}\left|\widehat{a}_{\ell}\right|^{2} & =-\ell^{2} \boldsymbol{\ell} \times \boldsymbol{m}\left(\widehat{\psi}_{k}^{*} \widehat{a}_{\ell} \widehat{a}_{m}+\widehat{\psi}_{k} \widehat{a}_{\ell}^{*} \widehat{a}_{m}^{*}\right), \\
\frac{\mathrm{d}}{\mathrm{d} t} m^{2}\left|\widehat{a}_{m}\right|^{2} & =m^{2} \boldsymbol{\ell} \times \boldsymbol{m}\left(\widehat{\psi}_{k}^{*} \widehat{a}_{\ell} \widehat{a}_{m}+\widehat{\psi}_{k} \widehat{a}_{\ell}^{*} \widehat{a}_{m}^{*}\right) .
\end{aligned}
$$

The right-hand sides of these equations sum up to zero, so the interaction within individual triads conserves energy as expected. Without loss of generality, let us assume $\ell<m$ in what follows.

Equations (3.1)-(3.3) fully describe the triad energetics and reveal a great deal about the nature of the energy transfer and dynamo action. In general, one magnetic mode gains energy at the expense of the other since the right-hand sides of (3.2) and (3.3) have opposite signs. Now suppose that the smaller-scale mode, i.e. mode $\boldsymbol{m}$, is the winner. In this case, the right-hand side of (3.1) is negative, implying a conversion from kinetic to magnetic energy (dynamo action). This is true whether $k>m$ or $k \leqslant m$ (including $k \leqslant \ell$ ) and establishes that the dynamo is accompanied by a direct magnetic energy flux. As far as the direct transfer of the total energy is concerned, the case $k>m$ corresponds to a relatively inefficient transfer, in the sense that the intermediate scale receives energy from both larger and smaller scales. Note that in 2D non-conducting fluids, transfer from the intermediate scale to both smaller and larger scales (or vice versa) is universal among interacting triads, nonetheless still giving rise to a net direct transfer. The case $k \leqslant m$ corresponds to a transfer of energy from the two larger scales to the third and smaller scale. This apparently more efficient transfer behaviour is characteristic of Burgers flows (Tran \& Dritschel 2010), for which velocity discontinuities develop in finite times, and has not been seen in other fluid systems. Thus, an efficient mechanism for direct energy transfer similar to that in Burgers flows is present in the 2D MHD equations. However, unlike the Burgers case, where each small scale can receive energy from larger ones to sustain a persistent direct energy flux, the energy of the mechanical mode $\widehat{\psi}_{\boldsymbol{k}}(t) \exp \{\mathrm{i} \boldsymbol{k} \cdot \boldsymbol{x}\}$ in the dynamo triads cannot be replenished in the same fashion. Instead, a mechanical small scale can be 'recharged' only through triad interactions that involve a return of energy from smaller scales to larger ones. This has a profound implication and is discussed presently. 
Suppose that in the above triad interaction, the larger-scale magnetic mode, i.e. mode $\boldsymbol{\ell}$, gains energy. This gain is at the expense of its smaller-scale counterpart $\boldsymbol{m}$, which also loses some energy to the mechanical mode $\boldsymbol{k}$ (the right-hand side of (3.1) is positive). Hence, an anti-dynamo is associated with an inverse magnetic energy flux. Similar to the above dynamo triad, this anti-dynamo triad can have either $k>m$ or $k \leqslant m$ (including $k \leqslant \ell$ ). The latter case corresponds to an inverse energy transfer from the smaller scale to both the intermediate and larger scales. The former case features a transfer of energy from the intermediate scale to both the larger and smaller scales. In both of these cases, the mechanical mode is energetically replenished, thereby making it possible for a persistent operation of the dynamo triads discussed in the preceding paragraph. Note that dynamo triads by themselves are able to excite the small scales magnetically but not mechanically. On the other hand, anti-dynamo triads by themselves cannot rid the large scales of any kinetic energy.

The analysis in the preceding paragraphs indicates that two distinct types of triads operate concurrently in the direct energy transfer. One of these is a dynamo while the other one is an anti-dynamo. The operation of dynamo triads relies on the recharging of their mechanical modes by anti-dynamo triads. While this does not guarantee dynamo saturation (or more accurately quasi-saturation) any better than the constraint due to energy conservation discussed above, it does provide another look at this widely observed phenomenon. Here 'quasi-saturation' means that $r(t)$ becomes quasi-steady and does not necessarily imply that the dynamo and anti-dynamo triads cease to be active. For high-resolution numerical analysis of anti-dynamo behaviour of both forced and unforced turbulence, see the recent studies of Loureiro et al. (2009) and Servidio et al. (2010).

\section{Numerical results}

We now present the results from a series of simulations, which support the above theories and reveal some rather unexpected features of the inertial range. Equations (2.6) and (2.7), where $v=\mu$, were integrated numerically using a pseudospectral method in a domain of side $2 \pi$. A fourth-order Runge-Kutta time-stepping procedure was used, with the dissipative terms incorporated exactly using integrating factors. The initial magnetic modes lay within the wavenumber range $[4,6]$ while the initial mechanical modes were confined to the range $[5,7]$. These modes were given random phases and equal magnitudes, and were then adjusted for a relatively broad range of initial magnetic-to-kinetic energy ratios $r_{0}$ while keeping a total energy of 2 . Five different values of $r_{0}$ were chosen: $r_{0}=1 / 16,1 / 4,1,4$ and 16 . For each value of $r_{0}$, three simulations were run at resolutions $1024 \times 1024,2048 \times 2048$ and $4096 \times 4096$, corresponding to $v=\mu=7.94 \times 10^{-4}, 3.15 \times 10^{-4}$ and $1.25 \times 10^{-4}$, respectively. Note that $v$ is decreased by a factor of $2^{4 / 3}$ when the resolution is doubled. This choice was made in view of recent theoretical results for turbulence with quadratic nonlinearity (Tran et al. 2011; Tran \& Blackbourn 2012; Tran \& Yu 2012) and turned out to provide adequate dissipation across resolutions once a 'correct' value of $v$ has been determined for the lowest one. For the five simulations at the highest resolution, the respective kinetic and magnetic Reynolds numbers $R e$ and $R m$, defined by

$$
\begin{aligned}
& R e=\frac{L^{4 / 3} \epsilon_{u}^{1 / 3}}{v}, \\
& R m=\frac{L^{4 / 3} \epsilon_{b}^{1 / 3}}{\mu},
\end{aligned}
$$



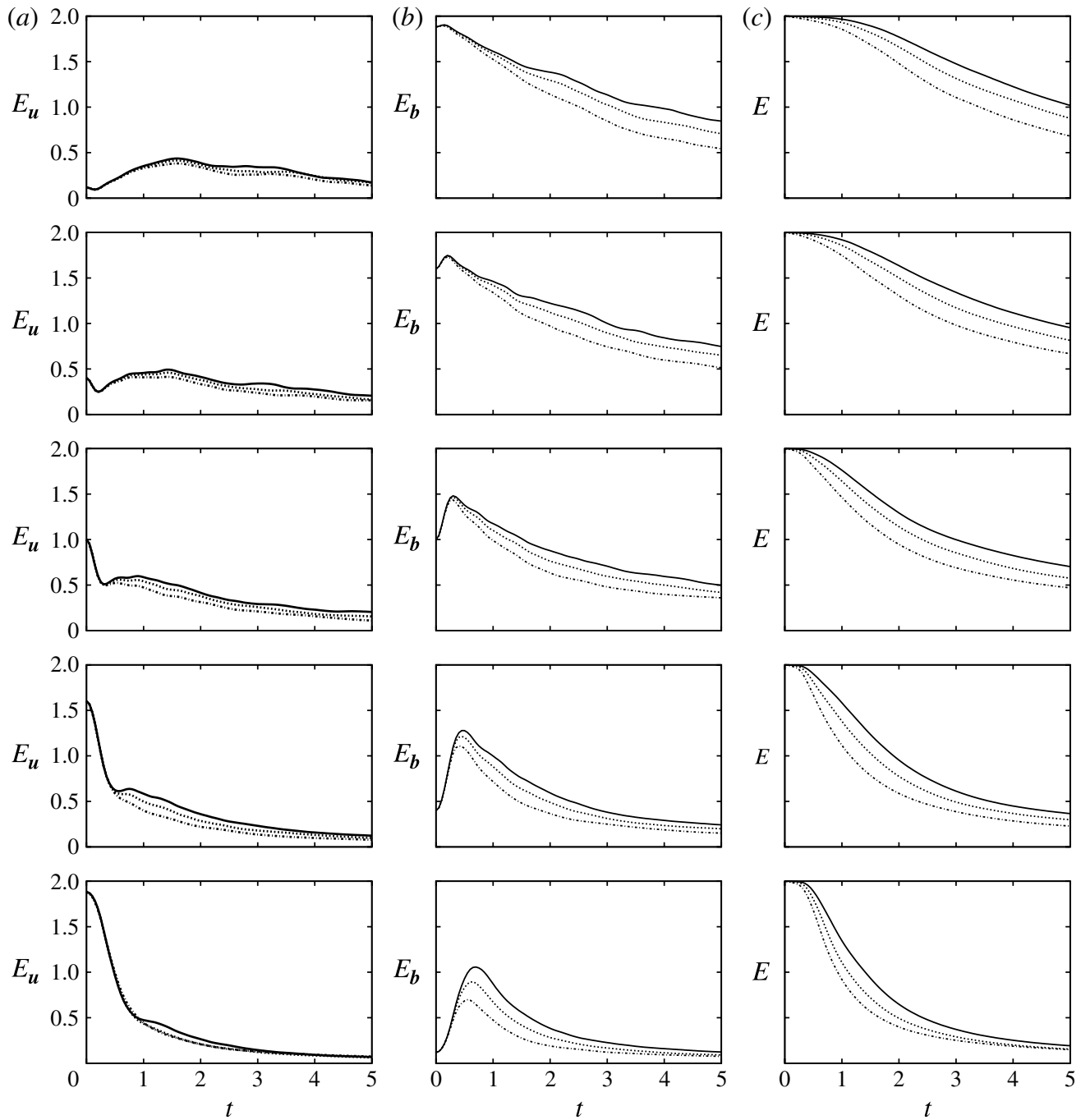

FIGURE 1. Kinetic $(a)$, magnetic $(b)$ and total $(c)$ energy versus $t$ for numerical simulations with resolutions $1024 \times 1024$ (dash-dotted), $2048 \times 2048$ (dotted) and $4096 \times 4096$ (solid). The rows are arranged in decreasing order of the initial magnetic-to-kinetic energy ratios $\left(r_{0}=16,4,1,1 / 4,1 / 16\right)$.

are approximately 68000 (at $t \approx T$ ). These dimensionless numbers emerge naturally from the mathematical analysis of Tran \& Yu (2012) (see also Tran \& Blackbourn 2012) and are virtually the same as their conventional counterparts defined in terms of the mean-square velocity $\left\langle|\boldsymbol{u}|^{2}\right\rangle^{1 / 2}$.

The evolution of the energy is documented in figures 1 and 2 . The former shows $E_{u}(t)(a), E_{b}(t)(b)$ and $E(t)(c)$ versus $t$ for the five sets of simulations described in the preceding paragraph and presented in decreasing order of $r_{0}$. The dash-dotted, dotted and solid lines represent the low-, middle- and high-resolution simulations, respectively. The latter depicts the time evolution of the energy ratio $r(t)=E_{\boldsymbol{b}}(t) / E_{\boldsymbol{u}}(t)$ for the highest-resolution runs. It can be seen that in the early stage, dynamo action takes place briefly for all $r_{0}$ (even for $r_{0}=16$, i.e. predominant magnetic energy), 


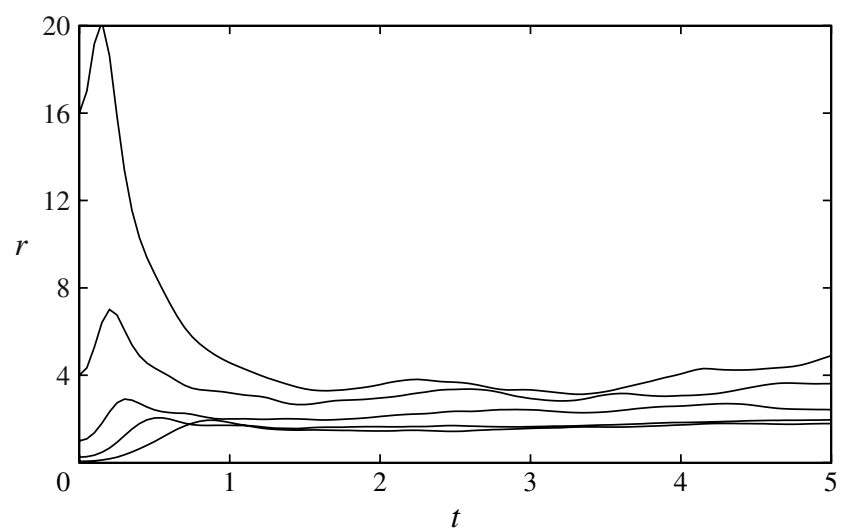

FIGURE 2. Magnetic-to-kinetic energy ratio $r(t)$ versus $t$ corresponding to the highestresolution simulations of figure 1. After the time of maximum dissipation, $r$ ranges approximately from 2 , for $r_{0}=1 / 16$, to 5 for $r_{0}=16$.
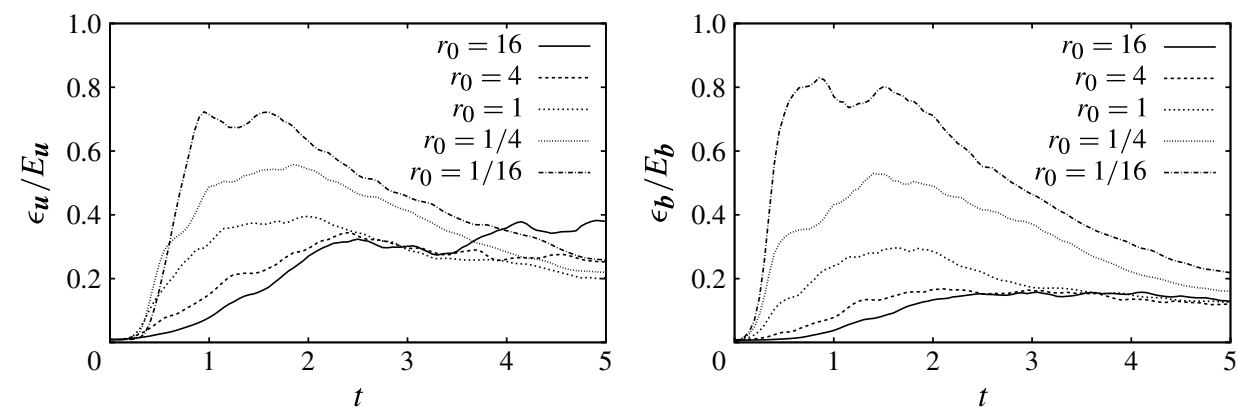

FIGURE 3. Exponential energy dissipation rates $\epsilon_{u} / E_{u}$ and $\epsilon_{b} / E_{b}$ versus $t$ for the five highest-resolution runs.

followed by anti-dynamo action. The cycle of dynamo and anti-dynamo then seems to repeat itself, more prominently for greater $r_{0}$, as can be recognized from the wavy appearance of the curves of $E_{b}(t)$ and $E_{u}(t)$. This is probably a signature of Alfvén waves, a detailed knowledge of which seems crucial for understanding MHD turbulence but is nonetheless not within the scope of the present study. In all cases, dynamo saturation occurs with $r \geqslant 2$ after a few cycles. This is consistent with a finding by Biskamp \& Welter (1989) for $r_{0}=1$, where a dynamo saturates at $r \approx 2$.

Figure 4 shows $\epsilon_{u}(t)(a), \epsilon_{b}(t)(b)$ and $\epsilon(t)(c)$ versus $t$ for the above five sets of simulations, laid out in the same decreasing order of $r_{0}$ as figure 1 . It can be seen across the board that energy loss through Ohmic dissipation is more than that through viscous dissipation, in agreement with previous studies for $\operatorname{Pr}=1$ by Haugen, Brandenburg \& Dobler (2003) and for $P r \leqslant 0.1$ by Brandenburg (2011). However, this is due mainly to the fact that the turbulence has more magnetic than kinetic energy $(r \geqslant 2)$ and does not necessarily imply a significant difference in the level of excitation of the mechanical and magnetic small scales. In fact, for all $r_{0}$ and $t \geqslant T$, it can be readily deduced from figure 3 that

$$
\frac{\epsilon_{u}}{E_{u}} \geqslant \frac{\epsilon_{b}}{E_{b}}
$$



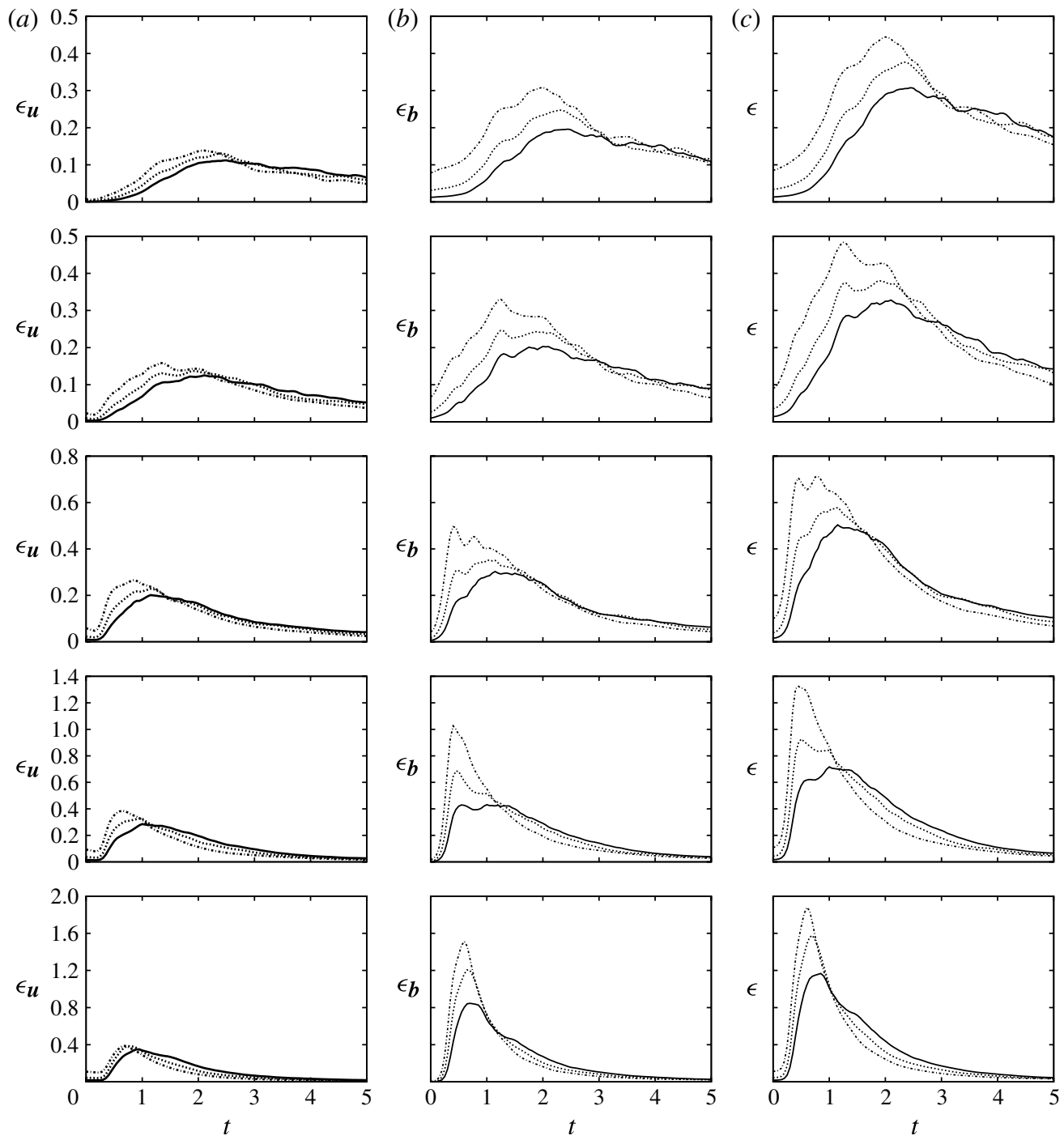

FIGURE 4. Kinetic (a), magnetic $(b)$ and total $(c)$ energy dissipation rates versus $t$ for the five sets of simulations described in figure 1. Again, the dash-dotted, dotted and solid curves correspond to the lower, intermediate and higher resolutions, respectively.

This implies that the exponential dissipation rate of magnetic energy is not greater than that of kinetic energy. Note that this behaviour may not hold for all $\mathrm{Pr}$, especially in the regime $\operatorname{Pr} \ll 1$ (Brandenburg 2011). The correlation between dynamo and direct magnetic energy flux manifests itself through the fact that a stronger dynamo (during the early stage) is accompanied by greater $\epsilon_{b}$, which peaks shortly after $E_{\boldsymbol{b}}$ achieves its global maximum (see the cases $r_{0} \leqslant 1$ of figures 1 and 2). An interesting feature is the more rapid decrease of $\epsilon_{T}$ for smaller $r_{0}$ when the resolution is increased. On this basis, one can anticipate that although $\epsilon_{T}$ is greater for smaller $r_{0}$ at the present resolution of $4096 \times 4096$, it may not necessarily be so at moderately higher resolutions. The interpretation is that the dynamo is very much an inertial-range 
(a)
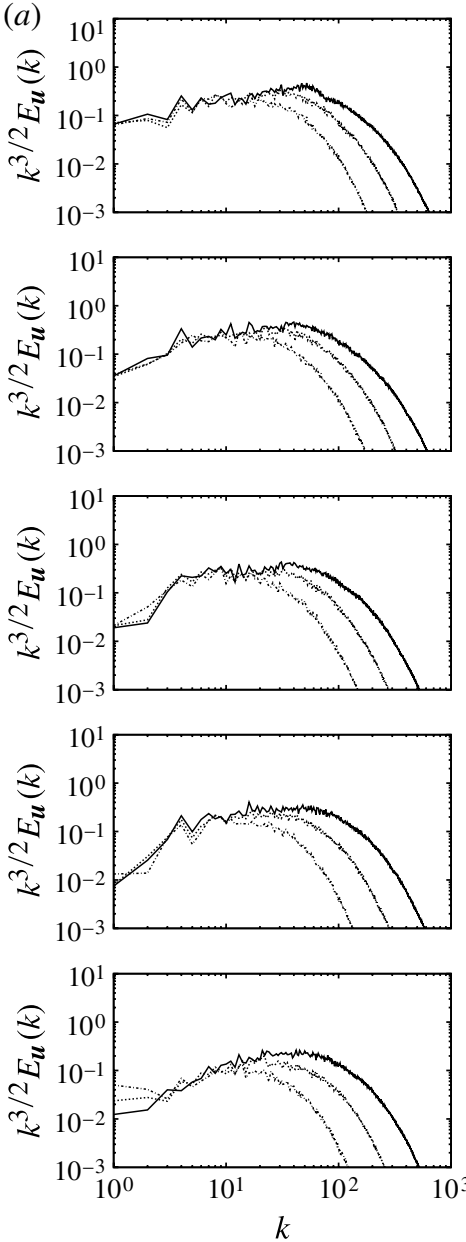
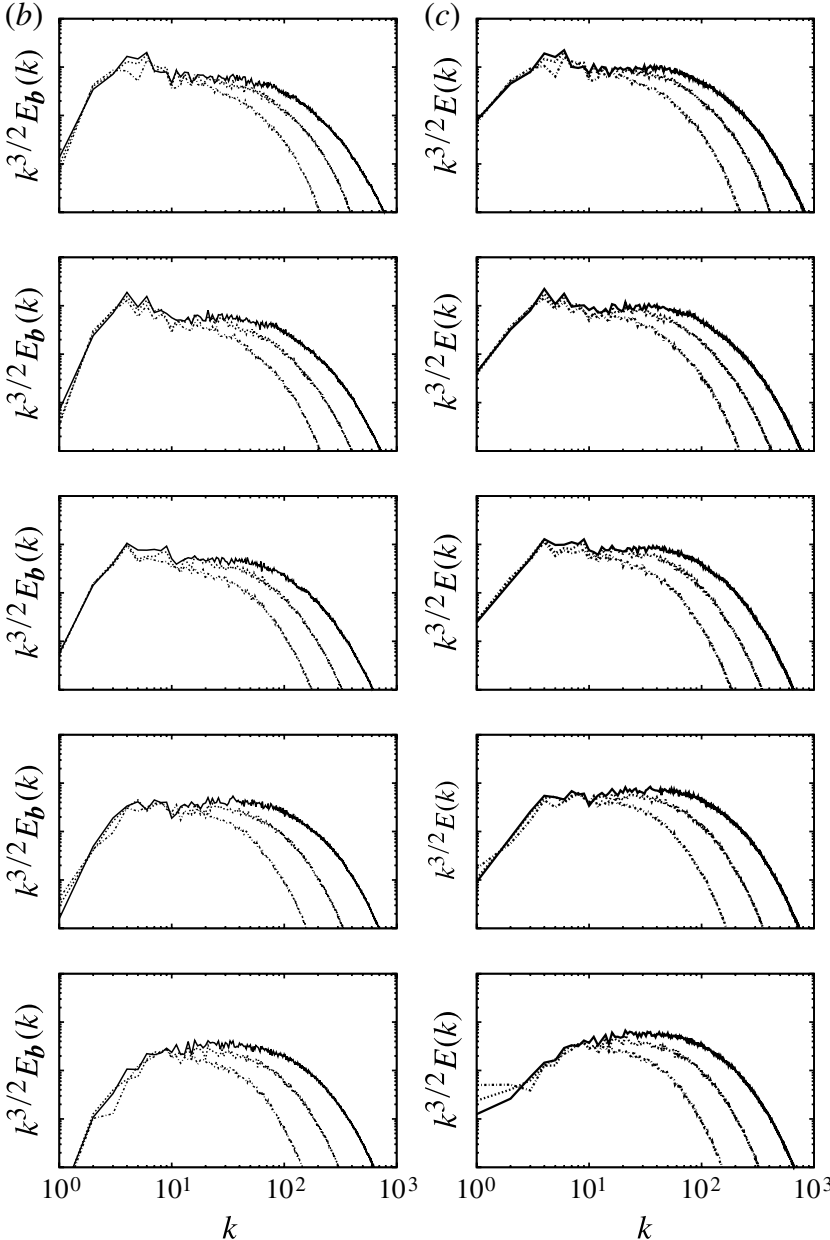

FIGURE 5. Kinetic (a), magnetic $(b)$ and total $(c)$ energy spectra, each multiplied by $k^{3 / 2}$, at $t=3.0$ for the simulations described in figure 1 .

phenomenon and that the turbulence is not necessarily more dissipative in the presence of a stronger dynamo.

The decrease of $\epsilon_{T}$, as well as of the global maxima of $\epsilon_{\boldsymbol{u}}(t)$ and $\epsilon_{\boldsymbol{b}}(t)$, as $v$ is decreased is accompanied by an increase of $T$. This allows the possibility of the divergence of $T$ and convergence of $\epsilon_{T}$ in the limit $v \rightarrow 0$. However, it is not known whether $\epsilon_{T}$ asymptotically vanishes or tends to a positive constant. This uncertainty can be appreciated by the fact that the 'tail' of $\epsilon(t)$ is higher for smaller $v$. In any case, the possible dependence of $T$ and $\epsilon_{T}$ on $v$ in the limit of small $v$ can be seen to be weak, probably in some logarithmic manner in $v$. These results have some far-reaching theoretical implications, which are discussed in the concluding section.

Figure 5 shows the kinetic energy spectrum $E_{u}(k)(a)$, magnetic energy spectrum $E_{\boldsymbol{b}}(k)(b)$ and total energy spectrum $E(k)(c)$, each multiplied by $k^{3 / 2}$, at $t=3$ for the above series of simulations. The dash-dotted, dotted and solid lines correspond to the lowest-, middle- and highest-resolution runs, respectively. Quite expectedly, within the inertial range (which approximately extends over one decade of wavenumbers for 
the highest-resolution case), these spectra become shallower for higher resolutions. However, their asymptotic behaviour, especially that of $E(k)$, as can be inferred from their shallowing tendency, may not be understood in terms of existing theories. At the highest resolution and for all $r_{0}, E_{\boldsymbol{u}}(k)$ is significantly shallower than $k^{-3 / 2}$ and clearly shallower than $E_{\boldsymbol{b}}(k)$, which is slightly shallower than $k^{-3 / 2}$ for $r_{0}=1 / 4,1 / 16$ and appears to be as shallow as $k^{-3 / 2}$ for $r_{0}=1,4,16$. The discrepancy in the slopes of $E_{\boldsymbol{u}}(k)$ and $E_{\boldsymbol{b}}(k)$ is strong evidence for no energy equipartition. The total energy spectrum $E(k)$ is already shallower than $k^{-3 / 2}$ for $r_{0}=1 / 4,1 / 16$ and can be expected to become shallower than $k^{-3 / 2}$ for $r_{0}=1,4,16$ at moderately higher resolutions. Hence, it is plausible that $E(k)$ is asymptotically shallower than the IK spectrum. The main questions raised are how much shallower $E(k)$ can become and whether or not its asymptotic slope is universal. These questions are beyond the scope of the present study, requiring a comprehensive numerical analysis and new theories (see some remarks in the final section).

In passing, it is noted that the lowest wavenumber $k=1$ is more strongly excited mechanically than magnetically, even for the case $r_{0}=16$ having predominant magnetic energy throughout: $r(t) \geqslant 4$. Given that the magnetic energy reservoir is closer to $k=1$ than the kinetic energy reservoir, one can infer that the inverse transfer of $\left\langle a^{2}\right\rangle$ is relatively weak, much weaker than the net inverse flow of $E_{\boldsymbol{u}}$ (net effect of inverse kinetic energy transfer by the vorticity advection term and direct total energy transfer). Hence, the concern over finite-size effects in numerical simulations of the present case is relatively minor compared with that of 2D Navier-Stokes turbulence.

Figure 6 shows the vorticity $(a)$ and electric current $(b)$ fields for the highestresolution runs at $t=3$. The greyscale vorticity field image was coloured between $-\|\omega\|_{\infty} / 2$ (black) and $\|\omega\|_{\infty} / 2$ (white) with $\omega<-\|\omega\|_{\infty} / 2$ equated to $-\|\omega\|_{\infty} / 2$ and $\omega>\|\omega\|_{\infty} / 2$ equated to $\|\omega\|_{\infty} / 2$. Here, $\|\omega\|_{\infty}$ denotes the maximum vorticity, whose evolution is described by figure 7. The image was adjusted in this way to enhance the resolution of moderate vorticity. Since the regions of exceedingly large $|\omega|$ are confined to very thin vortex filaments, this construction has virtually no noticeable effects on the structure of the vorticity field (except on resolution). The image of the electric current $j$ was treated in the same way, i.e. only $j$ within the range $\left[-\|j\|_{\infty} / 2,\|j\|_{\infty} / 2\right]$ was faithfully represented (see figure 7 for the evolution of $\|j\|_{\infty}$ ). The increase in small-scale features of the vorticity and current fields, particularly of the former, as $r_{0}$ is decreased, is consistent with the shallowing tendency of the spectra of figure 5. Finally, for a quantitative sense of the localness of exceedingly large values of $|\omega|$ and $|j|$, we note that for $t \geqslant T,\|\omega\|_{\infty}$ and $\|j\|_{\infty}$ are more than an order of magnitude greater than $\|\omega\|=\left\langle\omega^{2}\right\rangle^{1 / 2}$ and $\|j\|=\left\langle j^{2}\right\rangle^{1 / 2}$, respectively.

\section{Concluding remarks}

We have revisited the problem of energy transfer and inertial-range scaling in 2D MHD turbulence. The applicability of the IK theory, which was formulated for 3D MHD turbulence, to the present case has been examined both theoretically and numerically. While our results are consistent with the qualitative aspects of this theory on energy transfer reduction by Alfvén wave effects, its quantitative predictions of energy equipartition and $k^{-3 / 2}$ spectrum in the inertial range have been found to be unrealizable for fully developed turbulence satisfying the Kraichnan condition of magnetic energy at large scales exceeding total energy in the inertial range. More precisely, for turbulence at unity magnetic Prandtl number developed from a spectrally localized energy reservoir, the kinetic energy spectrum has been 
(a)
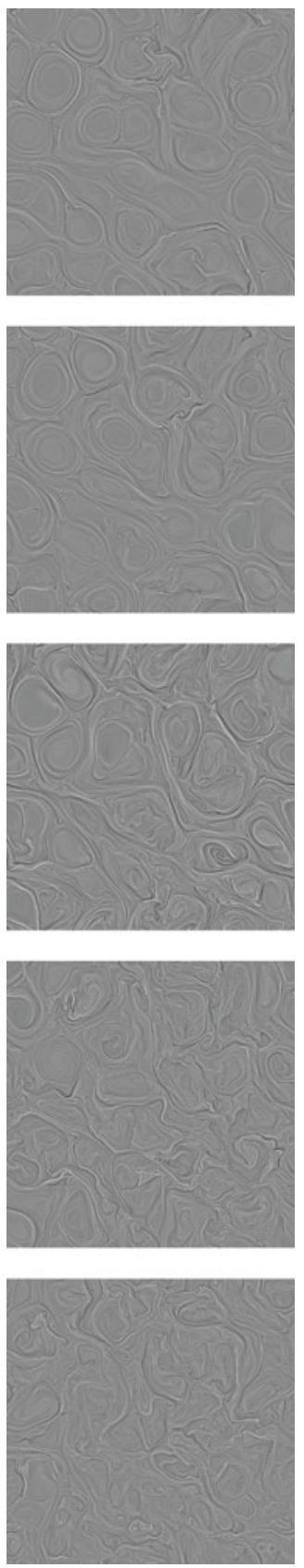

(b)
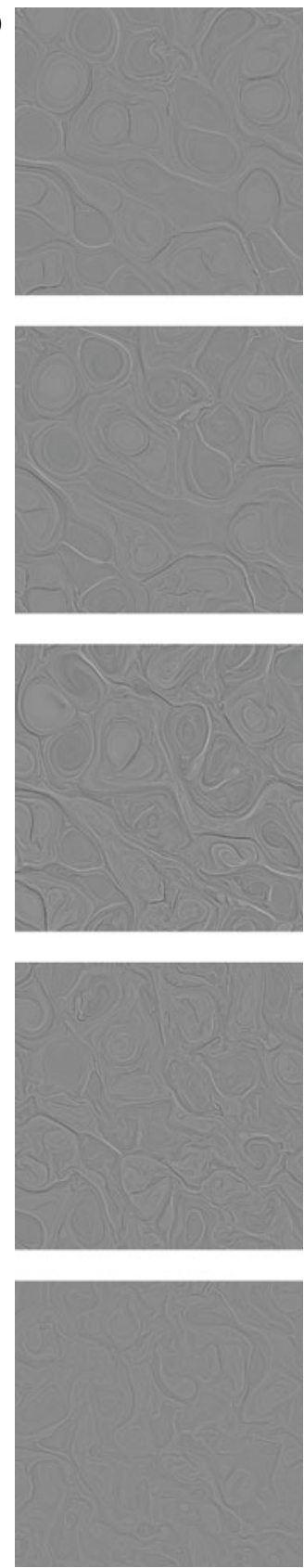

FIGURE 6. Adjusted vorticity $(a)$ and current $(b)$ fields corresponding to the spectra of the highest resolution of figure 5 , presented in the same order of decreasing $r_{0}$. Only $\omega \in\left[-\|\omega\|_{\infty} / 2,\|\omega\|_{\infty} / 2\right] \approx[-350,350]$ and $j \in\left[-\|j\|_{\infty} / 2,\|j\|_{\infty} / 2\right] \approx[-650,650]$ are faithfully represented. The values of each $\omega$ and $j$ outside the chosen interval are mapped to the nearer endpoint of the interval.

found to be significantly shallower than its magnetic counterpart, thereby suggesting no energy equipartition. Furthermore, the total energy spectrum has been observed 

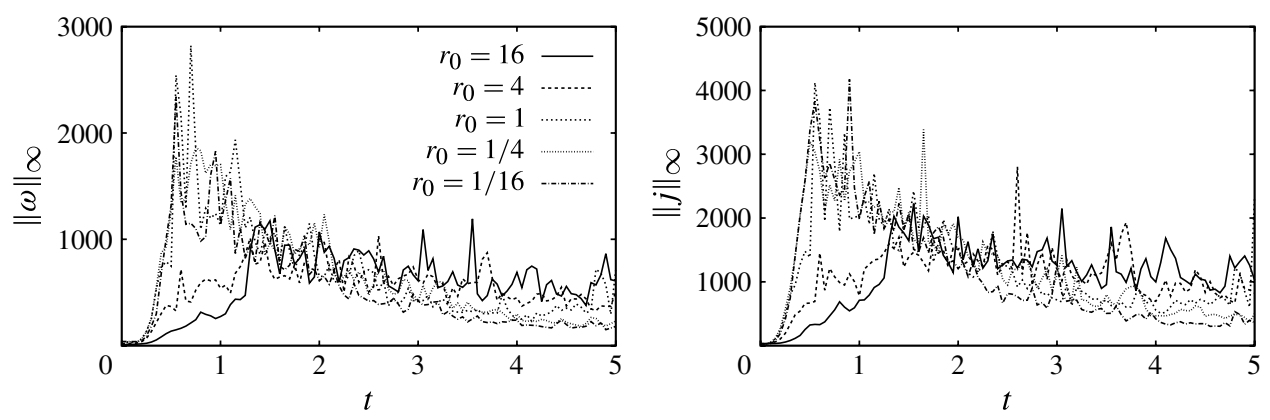

FIGURE 7. Maximum vorticity $\|\omega\|_{\infty}$ and current $\|j\|_{\infty}$ versus $t$ for the five highest-resolution runs.

to be shallower than $k^{-3 / 2}$, particularly for turbulence having moderate magnetic-tokinetic energy ratios (approximately 2). Further results from our numerical simulations suggest a weak dependence of the energy dissipation rate $\epsilon(t)$ on the viscosity $v$. In particular, as $v$ is decreased, the time $t=T$ of the peak energy dissipation rate $\epsilon_{T}=\epsilon(T)$ increases while $\epsilon_{T}$ decreases. Both the increase of $T$ and decrease of $\epsilon_{T}$ appear to be slow, probably logarithmic in $v$ in its small limit. This suggests the possibility of slow divergence of $T$ and (equally slow) convergence of $\epsilon_{T}$. However, it is not known whether $\epsilon_{T}$ would tend to a non-zero constant. Much higher resolutions than currently available to the present study are required to convincingly address this issue.

The IK theory was originally formulated for 3D MHD turbulence, where the notion of energy transfer reduction (or turbulence suppression) was apparently in reference to (and to be understood in terms of) the transfer of kinetic and magnetic energy in 3D without Alfvén wave effects. In the present context, this notion becomes largely irrelevant. The reason is that if the coupling between $\boldsymbol{u}$ and $\boldsymbol{b}$ via the Lorentz force were to be switched off, then the 2D kinetic energy would undergo an inverse transfer while its magnetic counterpart would be transferred to small scales through linear advection by a velocity field whose energy migrates the wrong way, to increasingly larger instead of smaller scales. The coupling between the velocity and magnetic fields acts through energy conversion to redirect the flow of kinetic energy. In other words, the dynamo and anti-dynamo are responsible for a direct kinetic energy flux. The important point to note here is that 2D MHD turbulence does not have the underpinning direct transfer of energy of the 3D case to fully make sense of Kraichnan's concept of transfer reduction. Hence, it is hardly surprising that the present results are not in line with the IK theory.

New theories and further numerical analysis are required to address the issue of inertial-range scaling of 2D MHD turbulence. This study has laid some of the groundwork for both. Improving the current numerical results could be as simple as extending the series of simulations in $\S 4$ to higher resolutions, with or without further broadening the range of $r_{0}$ and varying $\mathrm{Pr}$. In this regard, particular attention should be given to how the spectra shallow towards the 'ultimate' scaling $k^{-1}$, representing uniform distribution of energy among the wavenumber octaves. This distribution is realizable for some linear or nearly linear systems, such as passive-scalar transport by large-scale flows (Batchelor 1959; Tran 2008) and 2D Navier-Stokes turbulence (Tran, Dritschel \& Scott 2010), and, for the present case, is plausible if $T \rightarrow \infty$. The reason 
is that the divergence of $T$ means no excitation of ever-smaller dissipation scales in finite time - a manifestation of effectively linear behaviour of the small scales. The main question that a new theory may need to sort out is whether dynamo saturation represents a state of complete or nearly complete depletion of nonlinearity. Nearly linear small-scale dynamics would require good behaviour of the source terms in the following equations governing the vorticity $\omega$ and current $j$ :

$$
\begin{gathered}
\frac{\partial \omega}{\partial t}+J(\psi, \omega)=J(j, a)+v \Delta \omega, \\
\frac{\partial j}{\partial t}+J(\psi, j)=J(\omega, a)+2 J\left(\psi_{x}, a_{x}\right)+2 J\left(\psi_{y}, a_{y}\right)+\mu \Delta j .
\end{gathered}
$$

Now, for ideal dynamics $(v=\mu=0)$, the smoothness of either magnetic or velocity field implies that of the other and therefore of the system as a whole (Tran \& Yu 2012; Ohkitani 2006). Hence, in order for $T$ to diverge, it is sufficient to require good behaviour of the vorticity source term $J(j, a)$ alone. Here 'good behaviour' means that $J(j, a)$ does not diverge significantly more rapidly than $\omega$. A weaker and more tractable condition is $\langle\omega J(j, a)\rangle \leqslant\left\langle\omega^{2}\right\rangle \ln \left\langle\omega^{2}\right\rangle$, which allows for up to double exponential growth of $\left\langle\omega^{2}\right\rangle$. Note that our numerical results for $\epsilon_{u}(t)$ in $\S 4$ indicate much milder behaviour of $\left\langle\omega^{2}\right\rangle$ during the stage of most rapid growth. In handling this condition, one should watch out for possible high correlation between $\omega$ and $j$, in the sense that $\langle|J(\omega, j)|\rangle$ does not diverge so strongly. In particular, if $\langle|J(\omega, j)|\rangle$ does not diverge more strongly than $\left\langle\omega^{2}\right\rangle \ln \left\langle\omega^{2}\right\rangle$, then the required condition is satisfied since $\langle\omega J(j, a)\rangle=\langle a J(\omega, j)\rangle \leqslant\|a\|_{\infty}\langle|J(\omega, j)|\rangle$.

\section{Acknowledgement}

L.A.K.B was supported by an EPSRC post-graduate studentship.

\section{REFERENCES}

BATCHELOR, G. K. 1959 Small-scale variation of convected quantities like temperature in turbulent fluid. J. Fluid Mech. 5, 113-133.

Beresnyak, 2011 Spectral slope and Kolmogorov constant of MHD turbulence. Phys. Rev. Lett. 106, 075001.

BISKAMP, D. \& BREMER, U. 1993 Dynamics and statistics of inverse cascade processes in 2D magnetohydrodynamic turbulence. Phys. Rev. Lett. 72, 3819-3822.

Biskamp, D. \& Welter, H. 1989 Dynamics of decaying two-dimensional magnetohydrodynamic turbulence. Phys. Fluids B 1, 1964-1979.

Boldyrev, S. \& Perez, J. C. 2009 Spectrum of weak magnetohydrodynamic turbulence. Phys. Rev. Lett. 103, 225010.

Brandenburg, A. 2011 Nonlinear small-scale dynamos at low magnetic Prandtl numbers. Astrophys. J. 741, 92.

Cattaneo, F. \& Tobias, S. M. 2009 Dynamo properties of the turbulent velocity field of a saturated dynamo. J. Fluid Mech. 621, 205-214.

Dritschel, D. G., Scott, R. K., Macaskill, C., Gottwald, G. A. \& Tran, C. V. 2008 Unifying scaling theory for vortex dynamics in two-dimensional turbulence. Phys. Rev. Lett. 101, 094501.

Fyfe, D. \& Montgomery, D. 1976 High-beta turbulence in two-dimensional magnetohydrodynamics. J. Plasma Phys. 16, 181-191.

Galtier, S., Pouquet, A. \& MAngeney, A. 2005 Spectral scaling laws for incompressible anisotropic magnetohydrodynamic turbulence. Phys. Plasmas 12, 092310.

Goldreich, P. \& SRIDHAR, S. 1995 Toward a theory of interstellar turbulence. II. Strong Alfvénic turbulence. Astrophys. J. 438, 763-775. 
Grappin, R., Pouquet, A. \& Leorat, J. 1983 Dependence of MHD turbulence spectra on the velocity field-magnetic field correlation. Astron. Astrophys. 126, 51-58.

Haugen, N. E. L., Brandenburg, A. \& Dobler, W. 2003 Is nonhelical hydromagnetic turbulence peaked at small scales? Astrophys. J. 597, L141-L144.

Iroshnikov, P. S. 1964 Turbulence of a conducting fluid in a strong magnetic field. Sov. Astron. 7, $566-571$.

Kim, E.-J. \& DubRULle, B. 2002 Are the energy and magnetic potential cascades direct or inverse in 2D MHD turbulence. Physica D 165, 213-227.

Kraichnan, R. H. 1965 Inertial-range spectrum of hydromagnetic turbulence. Phys. Fluids 8 , 1385-1387.

Lee, E., Brachet, M. E., Pouquet, A., Mininni, P. D. \& Rosenberg, D. 2010 Lack of universality in decaying magnetohydrodynamic turbulence. Phys. Rev. E 81, 016318.

Loureiro, N. F., Uzdensky, D. A., Schekochinin, A. A., Cowley, S. C. \& Yousef, T. A. 2009 Turbulent magnetic reconnection in two dimensions. Mon. Not. R. Astron. Soc. 399, L146-L150.

Mininni, P. D., Pouquet, A. G. \& Montgomery, D. C. 2006 Small-scale structures in three-dimensional magnetohydrodynamic turbulence. Phys. Rev. Lett. 97, 244503.

MoffatT, K. H. 1967 On the suppression of turbulence by a uniform magnetic field. J. Fluid Mech. 28, 571-592.

MüLleR, W. \& GRAPPIN, R. 2005 Spectral energy dynamics in magnetohydrodynamic turbulence. Phys. Rev. Lett. 95, 114502.

Ng, C. S., Bhattacharjee, A., Munsi, D., Isenberg, P. A. \& Smith, C. W. 2010 Kolmogorov versus Irosnikov-Kraichnan spectra: consequence for ion heating in the solar wind. J. Geophys. Res. 115, A02101.

OhKitAni, K. 2006 A note on regularity conditions on ideal magnetohydrodynamic equations. Phys. Plasmas 13, 044504.

Podesta, J. J., Roberts, D. A. \& Goldstein, M. L. 2007 Spectral exponents of kinetic and magnetic energy spectra in solar wind turbulence. Astrophys. J. 664, 543-548.

Politano, H., Pouquet, A. \& Sulem, P. L. 1989 Inertial ranges and resistive instabilities in two-dimensional magnetohydrodynamic turbulence. Phys. Fluids B 1, 2330-2339.

Pouquet, A. 1978 On two-dimensional magnetohydrodynamic turbulence. J. Fluid Mech. 88, 1-16.

SRIDHAR, S. \& Goldreich, P. 1994 Toward a theory of interstellar turbulence. I. Weak Alfvénic turbulence. Astrophys. J. 432, 612-621.

Servidio, S., Matthaeus, W. H., Shay, M. A., Dmitruk, P., Cassak, P. A. \& Wan, M. 2010 Statistics of magnetic reconnection in two-dimensional magnetohydrodynamic turbulence. Phys. Plasmas 17, 032315.

Tessein, J. A., Smith, C. W., MacBride, B. T., Matthaeus, W. H., Forman, M. A. \& BOROVSKY, J. E. 2009 Spectral indices for multi-dimensional interplanetary turbulence at 1 AU. Astrophys. J. 692, 684-693.

Tobias, S. M. \& Cattaneo, F. 2008 Dynamo action in complex flows: the quick and the fast. J. Fluid Mech. 601, 101-122.

Tran, C. V. 2008 Local transfer and spectra of a diffusive field advected by large-scale imcompressible flows. Phys. Rev. E 78, 036310.

Tran, C. V. \& Blackbourn, L. A. K. 2012 A dynamical systems approach to fluid turbulence. Fluid Dyn. Res. 44, 031417.

Tran, C. V., Blackbourn, L. A. K. \& Scott, R. K. 2011 Number of degrees of freedom and energy spectrum of surface quasi-geostrophic turbulence. J. Fluid Mech. 684, 427-440.

Tran, C. V. \& Dritschel, D. G. 2006 Large-scale dynamics in two-dimensional Euler and surface quasigeostrophic flows. Phys. Fluids 18, 121703.

Tran, C. V. \& Dritschel, D. G. 2010 Energy dissipation and resolution of steep gradients in one-dimensional Burgers flows. Phys. Fluids 22, 037102.

Tran, C. V., Dritschel, D. G. \& ScOTT, R. K. 2010 Effective degrees of nonlinearity in a family of generalized models of two-dimensional turbulence. Phys. Rev. E 81, 016301. 
Tran, C. V. \& YU, X. 2012 Bounds for the number of degrees of freedom of magnetohydrodynamics turbulence in two and three dimensions. Phys. Rev. E (provisionally accepted).

Verma, M. K., Roberts, D. A., Goldstein, M. L., Gosh, S. \& Stribling, W. T. 1996 A numerical study of the nonlinear cascade of energy in magnetohydrodynamic turbulence. J. Geophys. Res. 101, 21619-21625. 